\title{
An Opportunistic Multiradio MAC Protocol in Multirate Wireless Ad Hoc Networks
}

\author{
Feng Chen, Hongqiang Zhai, and Yuguang Fang, Fellow, IEEE
}

\begin{abstract}
Multi-channel multi-radio technology offers a great space of resource diversity. In this paper, we propose an Opportunistic Multiradio MAC (OMMAC) protocol to improve system performance by utilizing multiradio diversity. The proposed OMMAC collects the physical layer feedback over multiple radios simultaneously and schedules multiple transmissions on the available channels accordingly. The channel based packet scheduling leverages the channel quality information to jointly select data rates, channels and packets to increase the overall spectral usage of multiple radios which have not been exploited in previous literature. Extensive results from both analysis and ns2 simulations demonstrate that OMMAC significantly improves the network throughput in both single- and multi-hop wireless multirate networks in terms of both aggregate and per-radio throughput.
\end{abstract}

Index Terms-Multiradio wireless networks, MAC protocol, diversity.

\section{INTRODUCTION}

I $\mathrm{N}$ recent years, industries have been putting a lot of efforts in investigating multi-channel multi-radio wireless networks [18][19][20]. The existence of multiple channels and multiple radios has greatly expanded manageable resource space, i.e., channel, radio, user, and time, to improve the network performance. The question of how to efficiently use the available resources in multiradio wireless networks is of great importance. In this paper, the proposed opportunistic multiradio MAC (OMMAC) is designed to exploit multi-radio diversity in order to enhance the throughput performance.

Opportunistic transmission schemes in general can be perceived as a way to utilize the physical-layer feedback from multiple sources to improve performance through medium access control [13], packet scheduling [12], and rate adaptation [1]. The proposed OMMAC can be regarded as a natural integration of these three approaches to maximize the extent of exploiting resource diversities. Not only responsible for granting medium access to packets, OMMAC also considers the total throughput of all radios on a node, which schedules

Manuscript received May 8, 2008; revised October 23, 2008; accepted December 15, 2008. The associate editor coordinating the review of this paper and approving it for publication was V. Leung.

F. Chen and Y. Fang are with the Department of Electrical \& Computer Engineering, University of Florida, Gainesville, Florida 32611. Y. Fang is also a Changjiang Scholar Chair Professor with National Key Laboratory of Integrated Services Networks, Xidian University, Xi'an, China (e-mail: \{chenf, fang\}@ecel.ufl.edu).

H. Zhai is with Philips Research North America, Briarcliff Manor, New York 10510 (e-mail: hong.zhai@philips.com).

This work was partially supported by the National Science Foundation under grants CNS-0721744 and DBI-0529012. The work of Fang was also partially supported by the 111 Project under B08038 with Xidian University, China.

Digital Object Identifier 10.1109/TWC.2009.080626 the transmission on per-channel basis, i.e., selecting the best transmission pair for each channel, rather than per-packet basis which selects the best channel for transmission every time a packet gains access to the medium [3]. Due to different interference level, channel fading statistics, and geographical difference of users, variable maximum data rates can be supported on different transmission links which is considered by OMMAC when optimizing the local spectral usage. The channel-based packet scheduling only takes advantage of the one-hop information, thus the routing and above layers remain unchanged. In order to exploit multi-radio diversity, OMMAC uses multi-cast RTS and virtual multi-CTS to collect receivermeasured channel quality information over several candidate transmission links. The above mentioned techniques make it possible to measure several channels simultaneously and send back a set of quality information at the same time. Multicast RTS and virtual multi-CTS are based on the RTS-CTS operation in IEEE 802.11, thus OMMAC does not generate exclusive communication overhead for extra control traffic compared to IEEE 802.11.

OMMAC is closely related to multi-channel multi-radio MAC design and MAC protocols exploiting resource diversities. Due to the technical limitation of the number of radios on each host, many previous works focus on how to efficiently use multiple channels with one or two transceivers[7][8][10][11][9]. None of them can be easily tailed to achieve the goal of scheduling multiple transmissions among several available radios on a single node. For practical multi-radio MAC schemes in multi-hop ad hoc wireless networks with the aim at improving network throughput, A. Adya et al.[4] proposed a multi-radio unification MAC protocol to coordinate the operation of multiple wireless network cards. However, the channel assignment is fixed, which limits the extent of using local spectrum. OMMAC pushes forward the idea of improving local spectral usage efficiency by considering the dynamic channel selection for several available radios simultaneously. It successfully coordinates multiple radios in utilizing the same channel pool and providing improved per-radio throughput by leveraging the multiradio diversity compared with previous research work such as OAR[1] and MOAR[3].

The remainder of this paper is organized as follows. First in Section II, we introduce the key ideas for OMMAC. Second in Section III, we describe the detailed protocol design issues. Third, we theoretically analyze the performance improvement of OMMAC in Section IV. The performance evaluation through ns2 is given in Section V. Finally, we draw the conclusion in Section VI. 


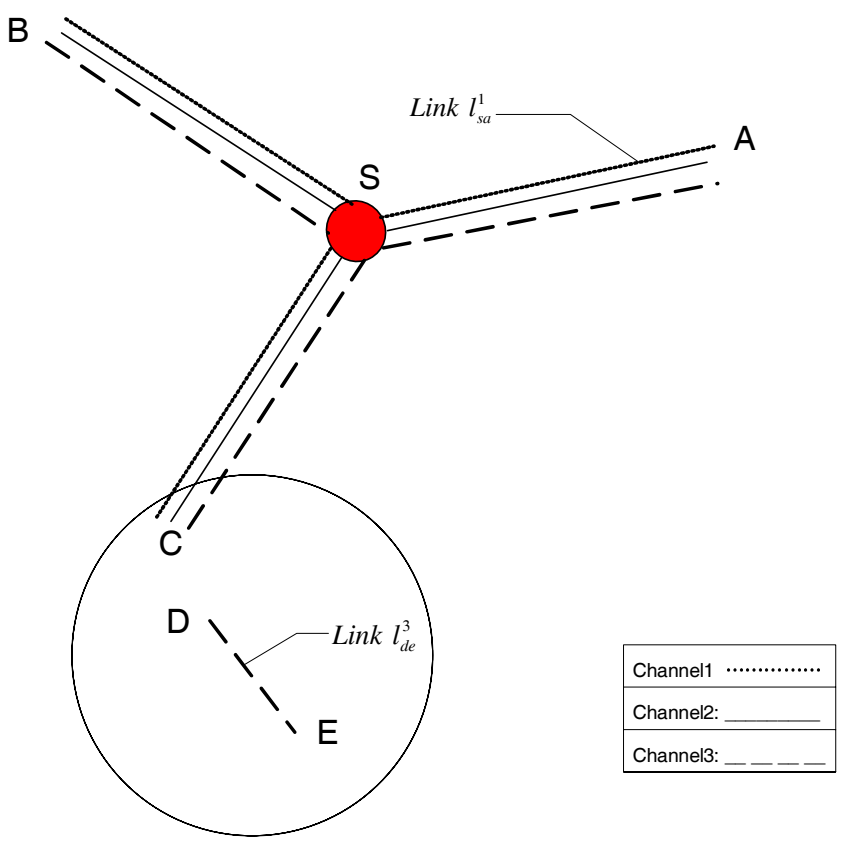

Fig. 1. Illustration for link diversity, channel diversity, and multi-radio diversity. Node $S$ is the source node, node $A$, node $B$, node $C$ are receivers. There is an ongoing transmission between node $D$ and node $E$ on channel 3 . The figure shows differences in geographical location and interference level which give rise to multiple diversities.

\section{DESIGN INSPRIATIONS}

\section{A. Multiple MAC Diversity Issues}

The multiple diversities come from geometrical difference among receivers, interference level and fading characteristics. We illustrate three kinds of diversities through the scenario shown in Fig. 1.

Link Diversity : Given a channel on a fixed frequency, the link diversity exists in the scenario in which there are several potential receivers for transmission. At a time, the quality of a link between a certain source and its next-hop neighbor is better than others. For example, the link set $\left\{l_{s a}^{i}, l_{s b}^{i}, l_{s c}^{i}\right\}$ can represent a source of link diversity, where $l_{s a}^{i}$ is defined as the link between $S$ and next-hop $A$ on channel $i$. We denote it as a link diversity set.

Channel Diversity: Given several available channels on orthogonal frequencies or codes, several transmissions can be carried out simultaneously on different channels without interfering with each other. For example, the link set $\left\{l_{s a}^{1}, l_{s a}^{2}\right.$, $\left.l_{s a}^{3}\right\}$ can represent a source of channel diversity. We denote it as a channel diversity set.

Multi-radio Diversity: Given several available channels and more than one radio on a single node, the multi-radio diversity arises in the scenario where different geographically located users experience different link qualities. For example, the link set $\left\{l_{s j}^{i}: j \in(a, b, c), i \in(1,2,3)\right\}$ can represent a source of multi-radio diversity. We denote it as a multi-radio diversity link set. From the definition, it is clear to see that the multiradio diversity set is the outer product of the link diversity set and the channel diversity set, thus providing a larger space for exploiting the diversities in the network.

For multi-channel multi-radio wireless networks, the freedom of exploiting different types of diversities has been

\begin{tabular}{|c|c|c|c|}
\hline Next hop Data rate & Channel 1 & Channel 2 & Channel 3 \\
\hline A & 36 & 24 & 24 \\
\hline B & 54 & 36 & 24 \\
\hline C & 36 & 54 & 6 \\
\hline
\end{tabular}

Fig. 2. Illustration for localized optimization. For each next-hop address (corrsponding to a packet), the link quality on different channel may be different. For each channel, the link quality with different receivers may be different. From Fig. 1, due to the ongoing transmission, the maximum supportable datarate on $l_{s c}^{3}$ is only 6 . The horizontal ellipse represents packetbased scheduling, while vertical ellipse represents channel-based scheduling.

greatly increased. Since several radios can work simultaneously on different channels, both link and channel diversity can be exploited simultaneously between a node and its neighboring nodes, which gives rise to multi-radio diversity.

\section{B. Channel-based Packet Scheduling}

In this section, we illustrate how OMMAC improves local spectral usage by considering the total throughput of locally available radios on a single node. One way to utilize the multi-radio diversity is to select a subset of the multi-radio diversity set to maximize local throughput with the limited number of available radios and channels. We denote the packet with the next hop address $A$ as $P_{a}$. Here the next-hop addresses also indicate their positions in the queue in an alphabetical order. Take Fig. 1 for instance. Suppose there are packets $\left\{P_{a}, P_{b}, P_{c}\right\}$ in the outgoing queue of node $S$. The current link qualities are described in Fig. 2. If $S$ selects the best channel whenever a packet gets the access for transmission, $S$ has to select link $l_{s a}^{1}, l_{s b}^{2}$ and $l_{s c}^{3}$ sequentially for packets $P_{a}, P_{b}$ and $P_{c}$. The total throughput for node $S$ at that time is $78 \mathrm{Mb} / \mathrm{s}$. We refer to it as packet-based packet scheduling. In OMMAC, with the objective of improving local spectral usage efficiency, the optimal packet scheduling policy would be the link subset $\left\{l_{s b}^{1}, l_{s c}^{2}, l_{s a}^{3}\right\}$ or $\left\{l_{s b}^{1}, l_{s c}^{2}, l_{s b}^{3}\right\}$. In this case, the total throughput is $132 \mathrm{Mb} / \mathrm{s}$. We call it channel-based packet scheduling. In Fig. 2, it is clear to see that channel-based packet scheduling is optimized for each channel, while packet-based packet scheduling is done for each packet. When applying the previous multi-channel schemes[2],[6],[7],[8],[10],[11] directly into multi-radio systems, they usually fall into the category of packet-based scheduling.

One of questions to be asked here is whether local throughput optimization helps improve aggregate throughput in multihop wireless network? It is believed that local optimization would not necessarily lead to the global optimization. However, solving global optimization of the throughput of network requires perfect knowledge of traffic pattern, network topology and interference distribution. Even with all the knowledge of the above, the problem itself is NP hard[4]. Thus, to our best knowledge, almost all the MAC protocols have not achieved the global optimal solution to the problem of 


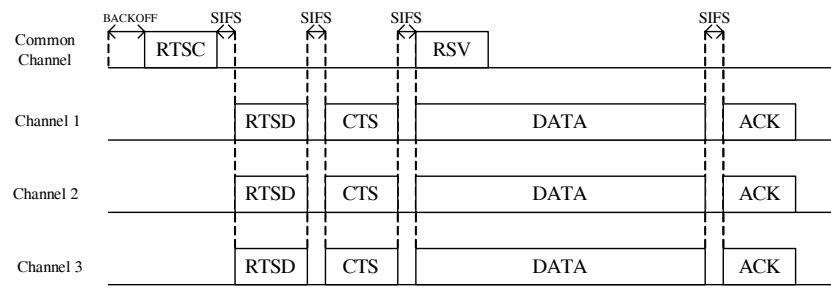

Fig. 3. Time line for OMMAC. Assume the current transmission uses 4 radios at one time. During the process of RTSC-RTSD-CTS, the sender is able to collect channel information on 9 links if the negotiation process is successful.

maximizing the throughput in wireless multihop networks. In some sense, all of the related research works such as [1][3][4] attempt to improve the local throughput instead. Many show the performance improvement in network throughput through experiments or simulations. We will show the performance evaluation of OMMAC in Sections IV and V.

\section{Opportunistic MAC using Multiple Radios}

From the above discussion, it should be clear now that in OMMAC the "opportunities" lie in the multi-radio diversity. The question of how to obtain the instantaneous link qualities in multi-radio diversity link set is the focus of design issues of OMMAC. OMMAC uses several available radios to multicast RTS on available channels to candidate receivers, coordinates receivers to simultaneously monitor several channels and send back CTS with as much information as possible. Using a single CTS to carry back the link quality information for several channels greatly enhances sender's ability to achieve the optimal scheduling according to the current link states. Moreover, it is worth pointing out that one RTS and CTS pair in IEEE 802.11 can only provide quality information on one link. Therefore, IEEE 802.11 needs much more pairs of RTS and CTS to collect the same information that OMMAC does.

\section{PROTOCOL DESCRIPTION}

In this section, we propose a novel Opportunistic Multiradio MAC (OMMAC) protocol to utilize the multi-radio diversity. Before elaborating the protocol in details, we first summarize our assumptions here. $N$ channels are available for use in a multi-hop multi-rate wireless network and all channels have the same bandwidth. None of the channels overlaps, so the packets transmitted on different channels do not interfere with each other. One of the channels is chosen to be the common channel. Each communication node is equipped with 1 to $M$ half-duplex transceivers. Every transceiver is capable of switching its channel dynamically and the channel switching time is 1us [5][3]. The transmission power is the same for each transmitter. We assume that channel is stationary across one successful packet transmission period [1].

\section{A. RTS on Common Channel(RTSC) and Multi-cast RTS on Data Channel(RTSD)}

RTSC is a frame sent on the common channel when a node gets access to the medium after backoff timer expires. RTSC informs candidate receivers in the transmitter's neighborhood to tune their radios to a set of specified channels. There are two important data fields in RTSC frame, the channel list and the next-hop list, which regulate receivers' behavior and establish communication channels between the transmitter and the receivers.

The channel list is constructed according to a local channel usage list. In OMMAC, each node maintains a local channel usage list which records the information which channel is available in the transmission range of the node. It also records the usage of radios. Let $C$ denote the total channel set in the network. Let $C_{a}$ denote the set of the local available channels. Let $R_{a}$ denote the set of the local available radios. The transmitter checks $C_{a}$ and $R_{a}$ when the backoff timer starts. Then $m$ channels are randomly selected by the transmitter from its available channel set $C_{a}$ to form the channel list in RTSC. The number of channels that the node will use for data transmission is at most $\min \left(\left|C_{a}\right|,\left|R_{a}\right|\right)$. Let $C_{t}$ denote the channels in the channel list.

The other important data field, the next hop list $H$, contains the addresses of candidate receivers. In order to fully exploit the multi-radio diversity as much as possible, the transmitter chooses as many packets with different next-hop addresses as possible under the constraint of the maximum number of packets to schedule is $\lceil\alpha m\rceil$. In case of $\alpha=1$, there is oneto-one mapping between the channels in the channel list and receivers in the next hop list. To be more specific, receivers which correctly receive the RTSD will determine their $C_{c t s}$ according to the channel list and next-hop list. For example, the third receiver in the channel list chooses the third channel in the channel list. In case of $\alpha>1$, there are several candidate receivers waiting on the same channel to send back CTS. For each receiver, the $C_{c t s}$ is determined by $R_{i} \% \mathrm{~m}$ where $R_{i}$ is the index of the receiver in the next-hop list, and $\%$ is the modulo operation which finds the remainder of division of $R_{i}$ by $\mathrm{m}$. The smaller index of the receiver in the next hop list is, the higher priority of sending back CTS the receiver has. The transmitter finishes the RTSD-CTS exchange process upon receiving the first CTS. In case of $\alpha<1$, there are more than one channels allocated for certain receivers. Each receiver identifies its first appearance in the next-hop list, and then using the rule in the case of $\alpha>1$. For the ease of exposition, we let $\alpha=1$ in the rest of the paper. If the same address has more than one position in RTSC's next-hop list, then CTS is sent on the channel with the smallest index in the channel list. The default channel used to send back CTS is denoted as $C_{c t s}$.

Upon receiving the RTSC, candidate receivers tune their available radios to a set of channels on the channel list. Denote the channel set that the $i_{t h}$ receiver in the next-hop list of RTSC is able to measure as $C_{r_{i}}$. Apparently, $C_{r_{i}} \subseteq C_{t}$. If the number of available radios at the $i_{t h}$ receiver, denoted as $M_{r_{i}}$, is equal to the length of the channel list in RTSC, then $C_{r_{i}}=$ $C_{t}$. Otherwise, receiver has to choose a subset of channels in $C_{t}$ to listen to. The first priority is given to $C_{c t s}$. The second priority is given to the channels that the available radios are already sitting on. For the rest of the available radios, the receiver randomly tunes the radios to channels which are in the channel list in RTSC.

RTSD is sent on the selected data channels according to the 
channel list after an SIFS interval following the transmission of RTSC. Each RTSD contains source address and the nexthop list which is the same in $R T S C$. RTSD is used as a probing message to enable the channel measurement at the candidate receivers. The multi-cast property of RTSD enables the measurement of channel quality at different receivers which may experience different link qualities on different channels or at different locations. Notice that each RTSD contains a copy of the next-hop list. In the case that a legitimate receiver may not hear RTSC but happen to hear RTSD on one or several data channels, the receiver will send back CTS according to RTSD. The channel list in RTSD will serve the same way as RTSC to enable the channel selection for sending back CTS.

By measuring the received signal strength of RTSD and estimating the channel noise level, the receiver can determine SNR which together with receiver sensitivity determines supportable data rate. The measured instant channel SNR remains valid within the coherence time. A conservative estimation about coherence interval given in [17], which are $51.98 \mathrm{~ms}$, $10.39 \mathrm{~ms}, 5.20 \mathrm{~ms}$ and $2.59 \mathrm{~ms}$ for mobile node with speeds of $1,5,10$ and $20 \mathrm{~m} / \mathrm{s}$, shows that coherence intervals are on the order of multiple packet transmission times which are 4 $\mathrm{ms}, 1.45 \mathrm{~ms}$ and $0.73 \mathrm{~ms}$ for a 1000 byte packet at 2, 5.5 and $11 \mathrm{Mbps}$. The fact that instant channel SNR remains valid within the coherence time helps to establish the rationality for including the receiver-selected data rate in CTS frame.

\section{B. Virtual Multi-CTS}

Virtual multi-CTS is a single CTS but containing the amount of quality information for several channels. CTS is sent after an SIFS upon receiving RTSD. The channel used to send back CTS, denoted as $C_{c t s}$, is indicated by $R T S C$. Since one receiver can listen to several channels simultaneously with multiple available radios, each receiver replies with a CTS frame containing the values of the maximal supportable data rate indices for channels in set $C_{r_{i}}$.

Even though we make sure that each receiver first tunes one of its available radios to $C_{c t s}$, it is still possible that the link quality on $C_{c t s}$ is so poor that RTSD cannot be heard. Then if it is the case, the receiver is going to skip channel $C_{c t s}$ and switch the radio to the $k$ th channel in the channel list in RTSC where $k=\left(C_{c t s}+1\right) \bmod \left|C_{t}\right|$. After a short sensing time $(20 \mu s)$, if the channel is idle, then the receiver will send CTS through that channel. Otherwise, it switches the radio to the $k$ th channel in the channel list in RTSC. The maximum number of channel switching is a parameter capturing the tradeoff between CTS waiting time and the amount of information the sender wishes to get. According to the channel switching rule here, receivers are coordinated to transmit on distinct channels even if they need to transmit CTS on the channels other than channel $C_{c t s}$. In case there is no successful RTSD-CTS exchange for a receiver on the data channel, the channel is not used for data transmission. The transmitter sends updated information about the availability of the channel on the common channel (see also in III-D) after timeout.

\section{Opportunistic Packet Scheduling for Multiple Radios}

Based on the information carried by CTS, a sender starts packet scheduling with the goal of optimizing local throughput of all its available radios. To put it in a straightforward way, the problem is formulated as below:

$$
\begin{aligned}
& \max _{i} \sum_{j}\left(\sum_{j} x_{j}^{i} q_{j}^{i}\right) \\
& \text { s.t. } \\
& \sum_{i} \sum_{j} x_{j}^{i} \leq m, \forall i \in C_{t}, j \in H, \\
& \sum_{i} x_{j}^{i} \leq\left|C_{r j}\right|, \forall j \in H, \\
& \sum_{j} x_{j}^{i} \leq 1, \forall i \in C_{t}, \\
& \sum_{i} x_{j}^{i} \leq y_{j}, \forall j \in H, \\
& x_{j}^{i} \in\{0,1\}, \forall i \in C_{s}, j \in H,
\end{aligned}
$$

where $q_{j}^{i}$ is the maximal supportable data rate on link $l_{s j}^{i}$ and $x_{j}^{i}$ is the packet scheduling policy for the $j_{t h}$ receiver on the $i_{t h}$ channel. The values of $x_{j}^{i}$ are either 0 or 1 , with 1 for scheduling the transmission on the $i_{t h}$ channel for packets to the $j_{t h}$ receiver in the next hop list. $y_{j}$ is the number of packets with the $j$ th address in the next hop list in the queue of the transmitter. $H$ is the next hop list in RTSC. Thus the objective function is to maximize the overall throughput of available radios at time $t$. The first constraint describes the fact that packet scheduling is confined by the number of available channels at the node. The second constraint comes from the requirement that the receiver can accept packets at one time up to the number of its own available resources $\left|C_{r i}\right|$, which is the number of channels the receiver is able to measure. The third constraint limits one transmission per channel at a time. The fourth constraint is that the number of the packets scheduled for the $j$ th receiver in the next hop list cannot exceed the number of packets with the $j$ th address in the queue of the transmitter.

The above formulation is a combinatorial optimization problem. An upper bound of this problem is using the vertices of $x_{j}^{i}=1$ for the maximal $q_{j}^{i}$ among all $j$ for each $i$, i.e., selecting the best link quality for each channel and scheduling the corresponding transmission with the maximal data rate $q_{j}^{i}$. Although the optimal solution may not be at the vertices of the above mentioned upper bound, the philosophy of solving the problem remains the same despite the limitation of the number of available channels, radios and packets. The opportunistic channel-based packet scheduling is carried out according to the solution of the above optimization problem. It is different from the approach many multi-channel schemes adopt, which is to select a relatively good channel for each transmission pair. As explained earlier in Section II-B, the approach we use here takes better usage of all available spectral usage.

Since the numbers of channels and radios are small, the recursive brute-force search can solve the problem in short 
time $^{1}$. The algorithm is executed by the sender to determine the packet scheduling strategy.

\section{Channel Reservation Frame and Data Transmission}

Channel ReSerVation frame (RSV) consists of channel usage field and channel utilization field. Channel usage field is set 1 as the channel is occupied by newly scheduled transmission. Channel utilization field is the length of the transmission on the channel. Carrying the information of the channel assignment and corresponding channel occupying time, RSV informs all the neighboring nodes that which channel is occupied and how long the channel is going to be occupied. In the meanwhile, data transmissions are scheduled on the selected data channels. The ACK is replied by the receiver on the data channel if the transmission is successful. Once the transmission of RSV ends, the common channel is again regarded idle. All the nodes update their local Channel Usage List according to RSV. Channel utilization field is used to set the network allocation vector (NAV) in the local channel usage list. The local channel usage list is updated whenever a channel is allocated for transmission or a NAV timer expires.

From the above, it should be clear that OMMAC is able to exploit multi-radio diversity as much as possible through multi-cast RTS, virtual multi-CTS, RSV, and local channelbased packet scheduling while keeping the communication overhead low.

\section{E. An Illustrative Example}

In this section, we outline the procedure of the OMMAC through an example as shown in Fig. 1. Suppose $S, A, B$, $C$ are the nodes with 3 available radios for data channels. In source $S$ 's outgoing queue, there are packets with next-hop addresses $A, B, C$. For $S$ to initiate a transmission, $S$ first multicasts a RTSC frame on the common channel. The RTSC frame includes the channel list indicating that $\operatorname{ch} 1, \operatorname{ch} 2, \operatorname{ch} 3$ are chosen from the available channels by $S$. It also includes the next-hop address list consisting of $A, B, C$ for the packets in its queue. Through the coordination of RTSC, nodes $A, B$ and $C$ switch their radios to listen to all these three channels ch $1, \operatorname{ch} 2$ and $\operatorname{ch} 3$. By listening to the following RTSD on the three data channels, $A$ is able to measure the instant channel qualities for link set $\left\{l_{s a}^{1}, l_{s a}^{2}, l_{s a}^{3}\right\}$ and sends back a single virtual multi-CTS with the channel qualities for several links on one of the channels among $\{\operatorname{ch} 1, \operatorname{ch} 2, \operatorname{ch} 3\}$. So do nodes $B$ and $C$. In this way, the link information collected by node $S$ could be in Fig. 2. As explained in Section II-B, the local spectral usage is maximized by carrying out the channel-based packet scheduling resulting in subsequent data transmission on the links $\left\{l_{s b}^{1}, l_{s c}^{2}, l_{s a}^{3}\right\}$ or $\left\{l_{s b}^{1}, l_{s c}^{2}, l_{s b}^{3}\right\}$. Then RSV is sent on the common channel to inform other nodes in the neighborhood to update their local channel usage lists. Since the receivers $A, B, C$ have already tuned their radios on channels indicated by RTSC, the reception of data packets can take place at the same time of transmitting RSV. The timeline for OMMAC is shown in Fig. 3.

\footnotetext{
${ }^{1}$ There are existing algorithms such as branch and bound algorithm which can be used to solve the problem more efficiently. The ways to solve such an optimization problem are out of scope of this paper
}

\section{F. Mechanism for Failure Transmission}

Let $T_{r t s c}, T_{r t s d}, T_{c t s}, T_{\text {data }}$, and $T_{a c k}$ be the transmission times for RTSC, RTSD, CTS, DATA, and ACK frames. $T_{\text {sifs }}$ denotes the time interval between consecutive frames, and $T_{\text {difs }}$ denotes the time interval for the backoff timer to start after the channel becomes idle. Upon successfully receiving the RTSC, the potential receivers should tune their radios on the corresponding available channels, while other nodes set their NAV to a default setting $T_{d c}$ for common channel and $T_{d t}$ for the possible transmission channels indicated in RTSC where $T_{d c}=T_{\text {sifs }} * 2+T_{r s t d}+T_{c t s}+T_{r s v}$ and $T_{d t}=T_{\text {sifs }} * 2+T_{r s t d}+T_{c t s}$.

If one or more RTSDs is heard on certain indicated channels, then $T_{d t}$ for each potential transmission channel at the receiver side is updated to $T_{\text {sifs }}+T_{c t s}$ and the potential receiver will send back CTS in the order indicated by RTSD. If none of the RTSDs is heard by a potential receivers (indicated by RTSC), $T_{d t}$ for each potential transmission channel at the receiver side is updated to $T_{s i f s}+T_{c t s}+T_{r s v}$ and the receiver will not send back CTS. Thus, in case of at least receiving one RTSD, the receiver can send back information about one or more channels. The more RTSDs the receiver gets, the more information it sends back to the sender. Missing all the RTSDs will results in no CTS sent back. According to RSV frame sent later by the sender on the common channel, the channel status of those potential transmission channels will be updated. Notice, all the other nodes who hear RSV on the common channel will make records of the status for all the transmission channels.

In case of the missing CTS from certain receiver, the information intended to be collected from that receiver is not available. Thus, the sender optimizes the transmission scheduling according to the rest of available information. Notice, the failure of a CTS from certain channel does not eliminate the possible usage of that channel. It only means the link quality from the sender to that receiver on that particular channel is not good for the moment. Other links may be able to use that channel due to the physical location variance.

The sender makes decisions about the optimal scheduling after timeout $T_{d t}$. For those channels which are chosen for current transmission, $T_{d t}$ is updated to $T_{\text {data }}+T_{\text {sifs }}+T_{a c k}$. For nodes who haven't heard RSTD, they are updated by RSV on common channels.

If a data transmission is failed, the data packet is not removed in the transmitting queue if the retransmission times for that packet do not exceed the retransmission limit. Thus, when the node gets to access the medium, it will consider the failed data together with other new data for schedule.

\section{Numerical ANALYSis}

In this section, we theoretically quantify the performance improvement through exploiting the multi-radio diversity. We model the time-varying wireless radio channels by considering both the effect of the path loss component and the small scale fading.

For the path loss component, the received power is given by:

$$
P_{r}(d)=P_{r}\left(d_{0}\right)\left(\frac{d_{0}}{d}\right)^{\gamma},
$$


where $d$ is the transmitter-receiver(T-R) separation distance, $d_{0}$ is the reference distance, $P_{r}\left(d_{0}\right)$ is the received power at $d_{0}$ and $\gamma$ is the path loss exponent with typical values $2 \leq \gamma \leq 5$.

For the small scale fading model, we use Ricean fading distribution for the received signal envelope $r$ whose probability density function is given by:

$$
p(r)= \begin{cases}\frac{r}{\sigma^{2}} e^{\frac{-\left(r^{2}+A^{2}\right)}{2 \sigma^{2}}} I_{0}\left(\frac{A_{r}}{\sigma^{2}}\right) & \text { for } \quad A \geq 0, r \geq 0 \\ 0 & \text { for } \quad r<0\end{cases}
$$

where $I_{0}(\bullet)$ is the modified Bessel function of the first kind and zero-order with $A$ as the peak amplitude of the dominant component. The Ricean distribution is often described in terms of a parameter $K$. It is defined as the ratio between deterministic signal power and the variance of the multiple paths, i.e., $K=\frac{A^{2}}{2 \sigma^{2}}$, where $\sigma^{2}$ is the time average power of the received signal before envelope detection.

\section{A. Case 1}

We first analyze the performance in the scenario where there is a single source $S$ with $N_{f}$ backlogged flows. Since there is no contention in such a network, we are able to focus on the performance gain coming from the multi-radio diversity. Suppose each node is equipped with $N_{r}$ radios. Let $m_{\left\{N_{f}, N_{r}\right\}}$ denote the minimum of $N_{f}$ and $N_{r}$. The throughput of each channel is:

$$
S=\frac{E(D A T A)}{E\left(T_{\text {backoff }}+T_{s}\right)},
$$

where

$$
T_{s}=T_{r t s c}+4 T_{\text {sifs }}+T_{r t s d}+T_{c t s}+T_{\text {data }}+T_{a c k}+T_{\text {difs } s} .
$$

$E\left(T_{\text {backoff }}\right)$ is the average backoff time and $E(D A T A)$ is the expected average length of data packet for each transmission. Recall $T_{r t s c}, T_{r t s d}, T_{c t s}, T_{\text {data }}$, and $T_{a c k}$ are the transmission times for RTSC, RTSD, CTS, DATA, and ACK frames. $T_{\text {sifs }}$ is the time interval between consecutive frames, and $T_{\text {difs }}$ is the time interval for the backoff timer to start after the channel becomes idle.

Clearly, the throughput for each channel depends on the value of $E(D A T A)$. Let $R$ denote the set of available data rates and $R=\left\{R_{i} \mid i=1, \ldots, N_{R}\right\}$ where $N_{R}$ is the number of available data rates. The larger the data rate is, the higher the index is. The corresponding Signal to Noise Ratio $(S N R)$ requirements for different data rates form the set $\left\{\beta_{i} \mid i=\right.$ $\left.1, \ldots, N_{R}\right\}$. Let $D$ denote the set of transmitter-receiver distances and $d_{j}$ denote the distance between the source and the $j_{\text {th }}$ receiver. We write $D=\left\{d_{j} \mid j=1,2, \ldots, N_{f}\right\}$. Let $r_{j}$ denote the received signal envelope of the received power at the $j_{t h}$ receiver. The $S N R$ at the $j_{t h}$ receiver is thus equal to

$$
S N R_{j}=\frac{P_{r}\left(d_{j}\right)}{P_{n}} r_{j},
$$

where $P_{n}$ is the noise power. The $S N R$ at each receiver is a random variable which follows the Ricean distribution. The set $S N R$ is formed by the $S N R$ value at the receiver, that is $S N R=\left\{S N R_{j} \mid 1 \leq j \leq m_{\left\{N_{f}, N_{r}\right\}}\right\}$. According to OMMAC, it endeavors to use the best link for each channel for data transmission. If the $S N R$ value of the best link on the channel should satisfy the $S N R$ requirement for $R_{i}$, the transmitter can adopt the data rate $R_{i}$ for the following data transmission. Let $\phi$ be the maximum value in the set $S N R$. The probability for the source of being able to transmit at the data rate $R_{i}$ is

$$
P\left(\phi \geq \beta_{i}\right)=1-\prod_{j=1}^{m_{\left\{N_{f}, N_{r}\right\}}} P\left(S N R_{j}<\beta_{i}\right)
$$

OMMAC uses the highest available data rate for transmission, thus the probability for using data rate $R_{i}$ by the source for each channel $P\left(R_{i}\right)$ is as follows:

$$
\begin{aligned}
P\left(R_{i}\right)= & P\left(\beta_{i+1}>\phi \geq \beta_{i}\right) \\
= & \prod_{j=1}^{m_{\left\{N_{f}, N_{r}\right\}} P\left(S N R_{j}<\beta_{i+1}\right)} \\
& -\prod_{j=1}^{m_{\left\{N_{f}, N_{r}\right\}}} P\left(S N R_{j}<\beta_{i}\right) \\
P\left(R_{N_{R}}\right)= & P\left(\phi \geq \beta_{\left.N_{R}\right)}\right. \\
& \forall 1 \leq i \leq N_{R}-1 \\
= & 1-\prod_{j=1}^{m_{\left\{N_{f}, N_{r}\right\}}} P\left(S N R_{j}<\beta_{N_{R}}\right) .
\end{aligned}
$$

If we consider the special topology where each receiver is equally separated, the density distribution function for $S N R$ at each receiver is identically independently distributed. In this case, $\widetilde{S N R}$ can also represent the value of $S N R$ at each receiver. Thus the above results can be given as:

$$
\begin{array}{r}
P\left(R_{i}\right)=P^{m_{\left\{N_{f}, N_{r}\right\}}}\left(\widetilde{S N R}<\beta_{i+1}\right) \\
-P^{m_{\left\{N_{f}, N_{r}\right\}}}\left(\widetilde{S N R}<\beta_{i}\right) \\
\forall 1 \leq i \leq N_{R}-1, \\
P\left(R_{N_{R}}\right)=1-P^{m_{\left\{N_{f}, N_{r}\right\}}\left(\widetilde{S N R}<\beta_{N_{R}}\right) .}
\end{array}
$$

The expected average transmission amount of data is:

$$
E(D A T A)=\sum_{i=1}^{N_{R}} R_{i} P\left(R_{i}\right)\left(T_{\text {data }}-T_{\text {preamble }}\right)-L_{H},
$$

where $T_{\text {preamble }}$ is the transmission time for the physical layer preamble of a DATA frame, and $L_{H}$ is the length of packet header which may include MAC, IP and TCP headers.

\section{B. Case 2}

In this section, we analyze the performance in the scenario where there are $N_{s}$ sources. There are $N_{c}$ channels available for use. Each source node has $N_{s}^{f}$ backlogged flows. Each node is equipped with $N_{r}$ radios. Let $m_{i}\left(1 \leq i \leq N_{s}\right)$ be the number of channels that the $i_{t h}$ source node will use if it successfully establishes the negotiation on the common channel. According to OMMAC, the $i_{t h}$ source node will use at most $\min \left\{N_{c}, N_{r}\right\}$ channels for data transmission at each time as long as there are enough available radios and channels. Clearly, $m_{i} \leq \min \left\{N_{c}, N_{r}\right\}$. 
Given the number of nodes $n$ contending for the transmission in wireless networks, the throughput for each channel can be obtained as follows:

$$
S=\frac{P_{s} P_{t r} E(D A T A)}{\left(1-P_{t r}\right) T_{e}+P_{t r} P_{s} T_{s}+P_{t r}\left(1-P_{s}\right) T_{c}},
$$

where

$$
P_{t r}=1-(1-\tau)^{n},
$$

and

$$
P_{s}=\frac{n \tau(1-\tau)^{n-1}}{1-(1-\tau)^{n}}
$$

Here, $T_{s}$ is the average successful transmission time, $T_{c}$ is the average collision time, $T_{e}$ is the duration of an empty slot time and equals to $20 \mu \mathrm{s}$.

$$
\begin{gathered}
T_{c}=T_{r t s c}+2 T_{\text {sifs }}+T_{r t s d}+T_{\text {ctsd }}+T_{\text {difs }} \\
T_{s}=T_{r t s c}+4 T_{\text {sifs }}+T_{r t s d}+T_{c t s d}+T_{\text {data }}+T_{a c k}+T_{\text {difs }}
\end{gathered}
$$

Each station transmits a packet with probability $\tau[16]$. Given the number of contending nodes $n$ in the wireless networks, $P_{t r}$ is the probability that there is at least one transmission in the considered slot time, $P_{s}$ is the probability that there is exactly one successful transmission on the channel.

The expected average amount of data transmission by using $m_{i}$ radios is $E\left(D A T A_{i}\right)$. Let $S_{i}$ be the throughput of the $i_{\text {th }}$ source on one channel. $S_{i}$ can be calculated by replacing $E(D A T A)$ with $E\left(D A T A_{i}\right)$ in Equation (13). The total throughput $S_{t}$ on all channels is equal to

$$
S_{t}=\sum_{i=1}^{|A|} S_{i} \times m_{i}
$$

where $A$ is a set which satisfies the following requirements:

$$
\sum_{i: a_{i} \in A} a_{i}=N_{c}
$$

In the above discussion, we assume that the data transmission is long enough to accommodate all $|A|$ contention periods. Otherwise, the control channel will be always busy while there are some idle data channels, which is also referred to as the common channel saturation problem.

In OMMAC, the common channel saturation problem is mostly overcomed by scheduling transmissions over several available channels at a time. It is enough to only accommodate about $N_{c} / N_{r}-1$ contention periods during a DATA transmission period. $N_{c} / N_{r}$ dramatically decreases with $N_{r}$. Moreover, we adopt the similar procedure of the burst transmission in OAR to have the same transmission time $T_{\text {data }}$ for all different rates. $T_{\text {data }}$ is set long enough for a transmission of a packet at the lowest rate.

\section{Numerical Results}

In this subsection, we use MATLAB to study the performance of OMMAC with different number of radios used for DATA frame transmission.
In the numerical analysis, for the path loss component, we use the free space propagation model when the transmitterreceiver distance is less than a crossover distance $d_{\text {crossover }}$, and the two-ray ground propagation model otherwise.

$$
P_{r}(d)= \begin{cases}\frac{P_{t} G_{t} G_{r} \lambda^{2}}{(4 \pi d)^{2} L}, & d \leq d_{\text {crossover }}, \\ \frac{P_{t} G_{t} G_{r} h_{t}^{2} h_{r}^{2}}{d^{4} L}, & d>d_{\text {crossover }},\end{cases}
$$

where both the transmitter antenna gain $G_{t}$ and the receiver antenna gain $G_{r}$ are set as one, the height of both the transmitter antenna $h_{t}$ and the receiver antenna $h_{r}$ are set as 1.5 meters, the system loss factor $L$ is set as one, the wavelength $\lambda=0.3282 m$ and $d_{\text {crossover }}=\frac{4 \pi h_{t} h_{r}}{\lambda}$. We study three rates $11,5.5$, and $2 \mathrm{Mbps}$. If only the path loss component is considered (no channel fading), their transmission radii are 100,200 , and $250 \mathrm{~m}$, respectively, by appropriately setting the $S N R$ requirements and the noise power. There are total 11 non-overlapping channels. In case 2 , the number of sources is 30 . We fix the transmitter and receiver distance as for the demonstration purpose. The minimum contention window size is $C W \min =31$. The rate for physical preamble frame is $R_{p l c p}=1$ Mbps. The basic rate used for control frames is $R_{\text {basic }}=2 \mathrm{Mbps}$. The number of radios used in the following experiments varies from 1 to 10 . The transmission time for RTSC, RTSD, CTSD, and ACK frame is calculated respectably as $T_{r t s c}=192 / R_{\text {plcp }}+(15+7 *$ numradio $) *$ $8 / R_{\text {basic }}, T_{r t s d}=192 / R_{\text {plcp }}+(15+7 *$ numradio $) * 8 / R_{\text {basic }}$, $T_{\text {ctsd }}=192 / R_{\text {plcp }}+(21+$ numradio $) * 8 / R_{\text {basic }}, T_{\text {ack }}=$ $192 / R_{\text {plcp }}+112 / R_{\text {basic }}$. The time intervals are $T_{\text {sifs }}=10 \mu \mathrm{s}$ and $T_{\text {difs }}=50 \mu \mathrm{s}$. The data transmission time is $T_{\text {data }}=$ $6000 \mu \mathrm{s}$.

Fig. 4 illustrates the throughput gain for case 2 when the number of radios for DATA frame transmission increases compared to the throughput with one data radio for DATA frame transmission. By exploiting the multi-radio diversity, the throughput increases along with the number of radios and is up to about $m$ times with $m$ radios for DATA frame transmissions. The $m$ times improvement occurs at distant receivers where the throughput is originally low. We only display the figure in the ranges from $0-300 \mathrm{~m}$. Within the range we are more interested, say less than 250 , normalized throughput is 1.8 times. We found that the figures for case 1 and case 2 are very similar and the throughput of case 2 is a little worse than that of case 1 due to the contention on the control channel. Here we only show the performance of case 2.

We also observe that the improvement brought by exploiting the multi-radio diversity occurs mainly at the transmitterreceiver distance where the maximum supported rate are more likely to change. This is similar to the improvement of OAR over the original 802.11. In addition, OMMAC is especially friendly to the users which have weak signals. The improvement for these users are huge. We also observe that the largest throughput gain per radio achieved by three radios. The increase of the normalized throughput gain gets smaller when the number of radio increases to be larger than 3 . It sheds some lights on the limitation of the performance gain in terms of the number of radios.

Fig. 5 and Fig. 6 show the absolute throughput for different number of radios when $K=1$. For case 1 , the source 


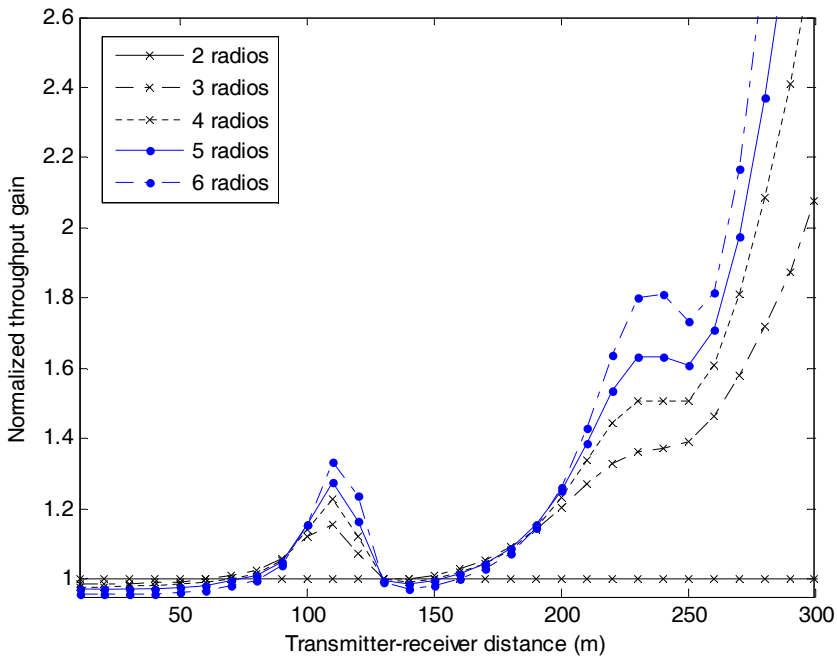

Fig. 4. Throughput gain normalized over the throughput of one data radio.

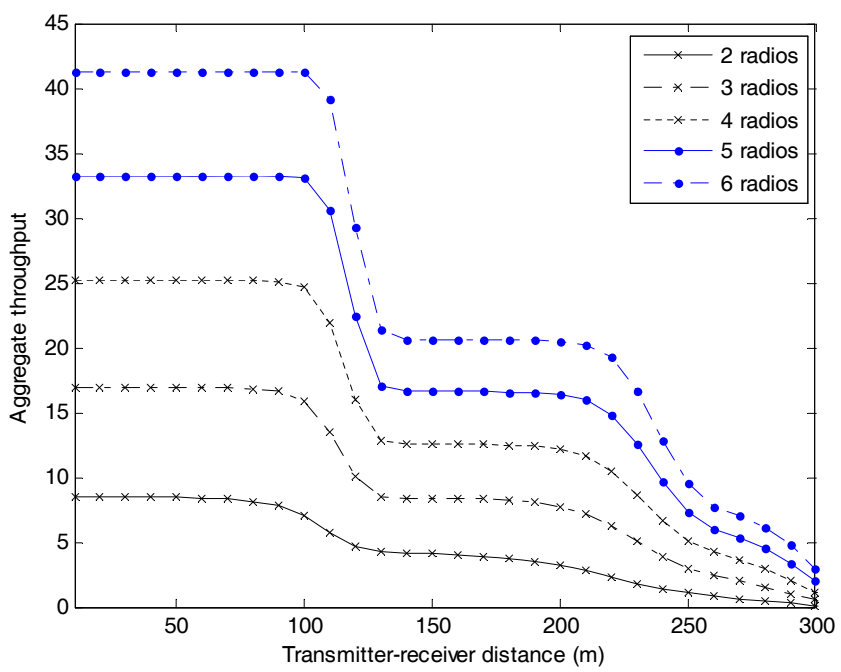

Fig. 5. Throughput of OMMAC in case 1.

node is able to use more available channels for simultaneous transmissions as the number of radios increases. For case 2 , several sources can share available channels and the total throughput is larger even with the same number of radios as case 1 . Thus, as long as the available channels are used up by the set of nodes, the total throughput is not increasing dramatically as the case 1 . However, OMMAC still manages to improve the performance especially at the distance where the maximum supported rate are more likely to change which is consistent with Fig. 4.

Besides the above observations, it clearly demonstrates that OMMAC extends the transmission range of each rate by exploiting the multi-radio diversity. This is really important in multihop networks, where routing algorithms fight to find the optimum tradeoff between the rates and the communication distances when choosing forwarding nodes [15].

\section{Simulation Results}

In this section, we use ns2 simulator to evaluate the performance of OMMAC. We extend ns2 to support multiple rates as in[14] and the Ricean propagation model as in MOAR[3].

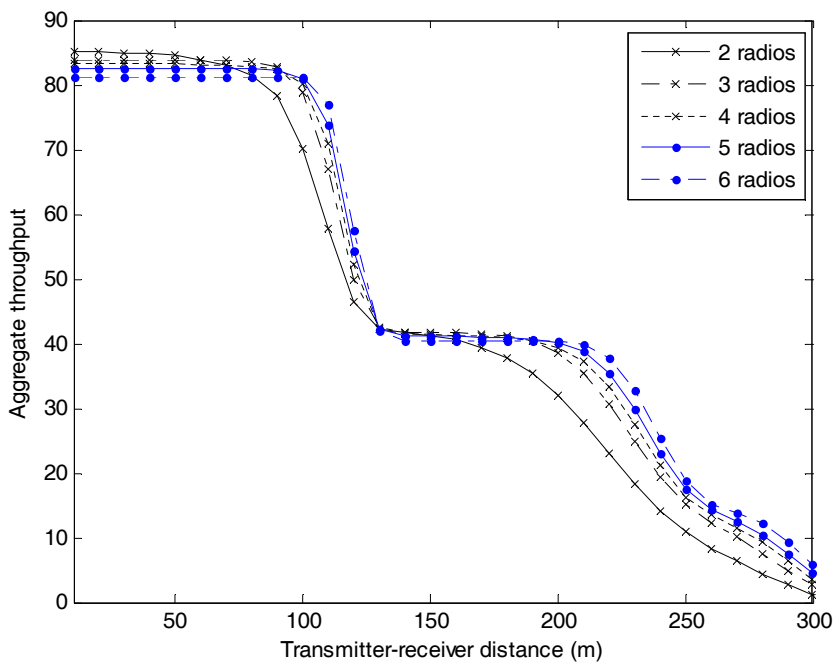

Fig. 6. Throughput of OMMAC in case 2 .

First, we begin with single-hop topologies to study the main protocol properties and illustrate the impact of different parameters - the number of radios and the number of flows-on throughput performance. Second, we study multi-hop topologies to verify the performance in wireless ad hoc network. We also compare the aggregate throughput performance of OMMAC with that of MOAR[3] and demonstrate that OMMAC significantly improves the throughput by exploiting the multiradio diversity. Moreover, we show the per radio throughput of OMMAC which also outperforms OAR and MOAR. We denote this throughput as "per radio" throughput in the figures. In the simulation, there are total 11 non-overlapping channels. Three different rates, 2, 5.5, and $11 \mathrm{Mbps}$, are studied. As in MOAR, their default transmission radii are 250, 200, and $100 \mathrm{~m}$, respectively, and may change due to channel fading. UDP traffic is used. Simulation time is 50 seconds in each run.

\section{A. Single-hop rectangular topology}

In the single-hop topology, all nodes are within the transmission range of each others. To separate the influence of topology, we start with a $200 m \times 200 m$ rectangular topology to study the impact of different parameters on the performance. There are four nodes on each extreme point of the rectangle and four nodes on the middle of each edge of the rectangle. The source node is located on the central point of the rectangle. We change the parameters such as the number of radios, the number of flows, and the channel fading parameter in the simulation to observe their impact on throughput.

Impact of the number of radios: Here we use different number of radios to investigate the relationship between the number of radios and the throughput. There are eight flows generated from the source node $S$ to the other eight nodes. Simulation results show that the throughput increases along with the number of radios. This indicates that OMMAC has successfully coordinated the available radios for transmission to improve the local spectral usage. The aggregate throughput does not go all the way up but it is limited to the number of available radios or channels. The aggregate throughput 


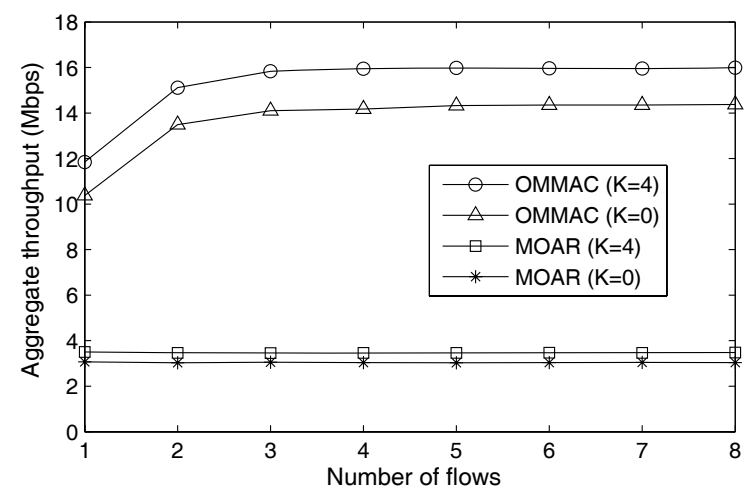

Fig. 7. Aggregate throughput of OMMAC as a function of number of flows with different Ricean parameters compared with that of MOAR in the singlehop rectangular topology.

remains steady when the number of radios exceeds the number of flows. If the number of available channels is smaller than the number of radios, we can expect the similar result.

Impact of the number of flows: In this simulation, we set the number of radios as four. From Fig. 7, we see a dramatic increase in throughput when the number of flows increases from 1 to 2 , which results from the unique feature of packet scheduling in OMMAC. When the outgoing queue has many packets with different next-hop addresses, the multiradio diversity consists of a large set of link diversities, which gives OMMAC the opportunity to optimally schedule the transmissions and improve the throughput. Due to the limitation of the extent of channel quality variation and the number of radios, the throughput becomes steady when the number of flows is close to the number of radios.

We further show in Fig. 8 that the per-radio throughput of OMMAC is greater than that of MOAR even though for each radio OMMAC has averagely fewer channels to schedule the transmissions than MOAR, the multi-radio diversity still offers performance improvement in aggregate per-radio throughput in OMMAC. When the number of flows is 1 , the per radio throughput of OMMAC is less than that of MOAR is mainly because of the communication overhead of OMMAC. As the increase of the number of flows, the multiradio diversity is greatly increased. The higher per-radio performance of MOAR shows that OMMAC has efficiently utilized the multiradio diversity despite the communication overhead.

Impact of the Ricean parameter $K$ : Here we use four radios in each node. There are still eight flows generated from the source $S$ to the other eight nodes. We tune the Ricean parameter $K$ from 0 to 6 . The larger the value of $K$ is, the smaller variation there is in channel quality. Fig. 9 shows throughput of both MOAR and OMMAC. Here the aggregate per-radio throughput in OMMAC is still greater than MOAR. Similar with MOAR, OMMAC's throughput increases when $K$ increases.

\section{B. Single-hop random topology}

In this subsection, we consider a random topology where the mobile nodes are uniformly distributed in a circular area with a diameter of $250 \mathrm{~m}$. We choose Ricean fading parameter $K$

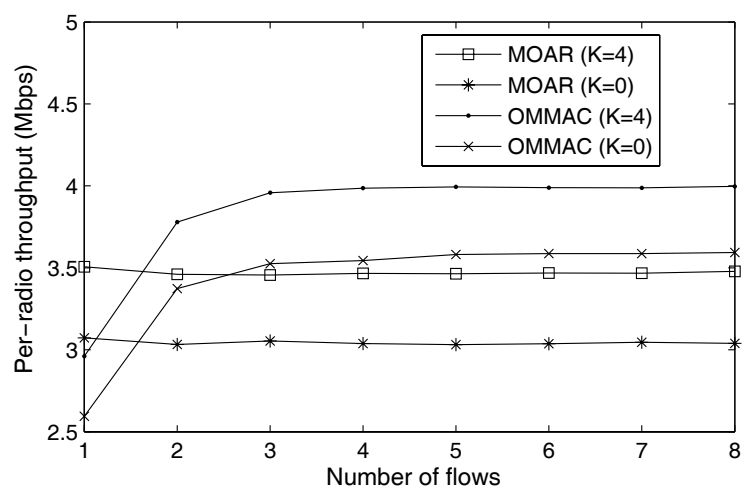

Fig. 8. Per radio throughput of OMMAC as a function of number of flows with different Ricean parameters compared with that of MOAR in the singlehop rectangular topology.

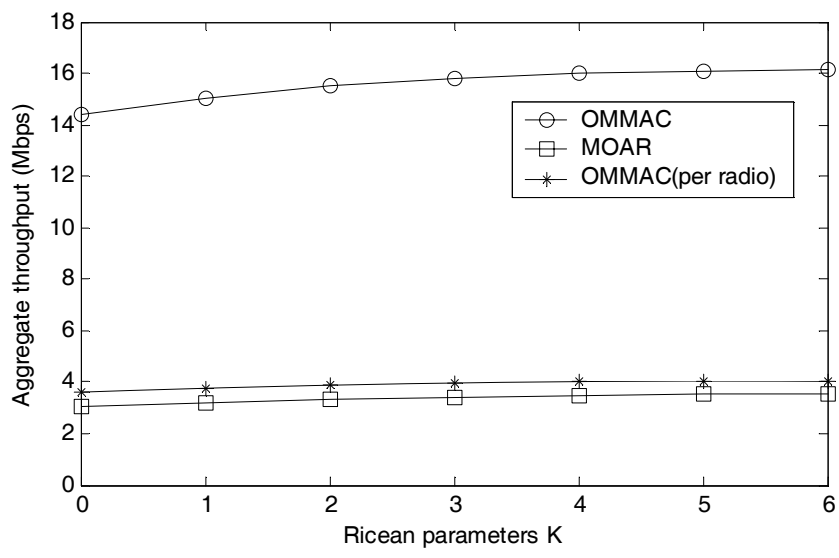

Fig. 9. Throughput of OMMAC as a function of the Ricean parameter $\mathrm{K}$ in the single-hop rectangular topology.

equal to 4 . There are 4 radios in each node. The throughput of both MOAR and OMMAC is shown in Fig. 10. The increase in aggregate throughput along with the number of flows again shows the benefit of exploiting multi-radio diversity. OMMAC improves the throughput by 2.33 times up to 8.91 times. Moreover, the aggregate per-radio throughput in OMMAC outperforms MOAR by average 1.4 times.

\section{Multi-hop random topology}

In this subsection, we study a multi-hop topology where mobile nodes are uniformly distributed in a $1000 \mathrm{~m} \times 1000 \mathrm{~m}$ rectangular area. We choose Ricean fading parameter $K$ equal to 4 . There are 4 radios in each node. We use AODV as the routing protocol. As shown in Fig. 11, OMMAC significantly improves the throughput compared with MOAR, and the gain is from 3.27 times to 11.22 times when the number of flows increases from 2 to 16 . Also the aggregate per-radio throughput in OMMAC improves as the number of flows increase and outperforms MOAR when the number of flows is greater than 4 . The first flow has zero throughput since they are disconnected in the simulated random topology.

From all the results above, we can see that OMMAC performs well in exploiting the multi-radio diversity. By exploiting channel diversity over multiple channels, MOAR improves throughput by up to $60 \%$ [3] compared to OAR. 


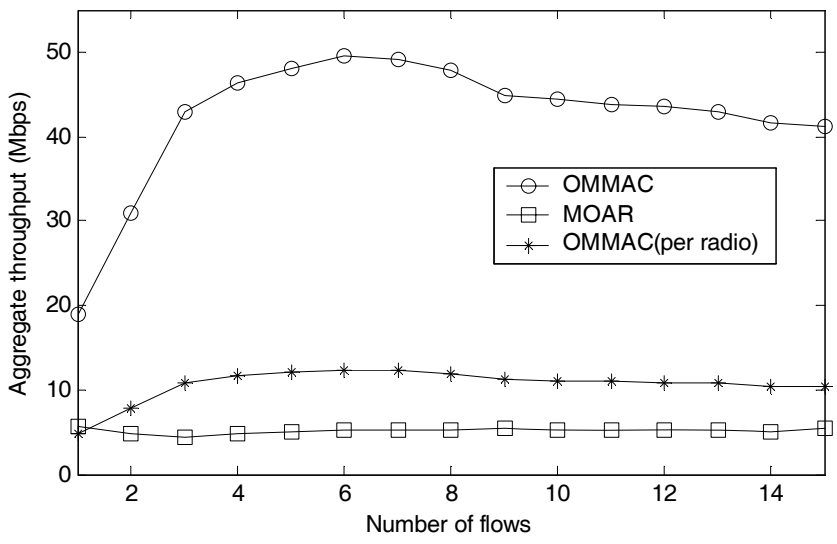

Fig. 10. Throughput of OMMAC as a function of number of flows in the single hop random topology in comparison with that of MOAR.

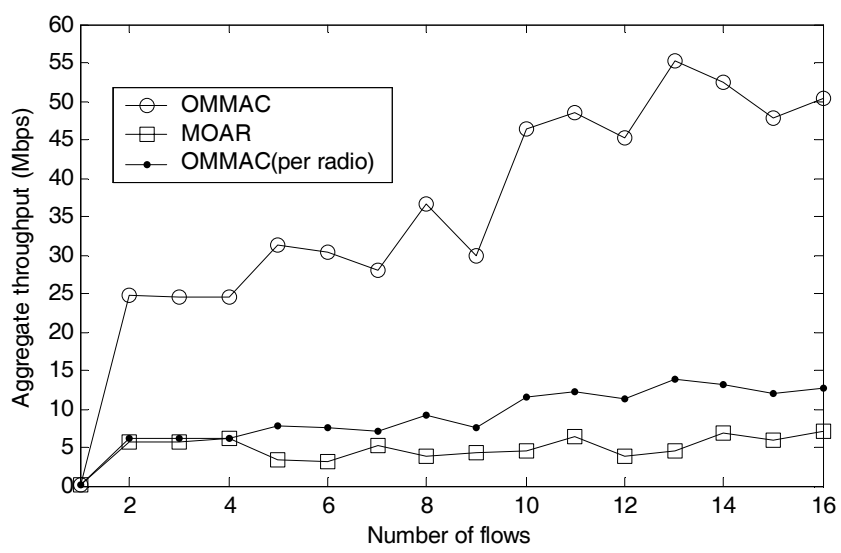

Fig. 11. Throughput of OMMAC as a function of number of flows in the multi-hop random topology in comparison with that of MOAR.

By exploiting the multi-radio diversity over multiple radios and channels, OMMAC further improves both multi-radio and aggregate per-radio throughput.

\section{CONCLUSION}

In this paper, we propose a novel opportunistic multiradio MAC as an efficient way of exploiting multi-radio diversity through multi-cast RTSD, virtual multi-CTS and channelbased packet scheduling. The existence of multi-radio diversity provides a larger space for selecting links with good channel quality for transmissions. OMMAC plays great endeavor in coordinating multiple radios on each node to gather link quality information upon which it carries the channel-based packet scheduling by considering the total throughput of available radios. Both numerical and ns2 simulation results have shown that OMMAC has significantly improved not only the aggregate but also the per-radio throughput performance.

\section{REFERENCES}

[1] B. Sadeghi, V. Kanodia, A. Sabharwal, and E. Knightly, "OAR: an opportunistic auto-rate media access protocol for ad hoc networks," Wireless Networks, vol. 11, no. 1-2, pp. 39-53, Jan. 2005.

[2] J. Mo, H. W. So, and J. Walrand, "Comparison of multi-channel MAC protocols," in Proc. International Workshop Modeling Analysis Simulation Wireless Mobile Systems, 2005.

[3] V. Kanodia, A. Sabharwal, and E. Knightly,"MOAR: a multi-channel opportunistic auto-rate media access protocol for ad hoc networks," in Proc. IEEE International Conf Focusing Broadb Commun., Networks, Systems (BROADNETS'04), 2004.

[4] A. Adya, P. Bahl, J. Padhye, A. Wolman, and L. Zhou, "A multi-radio unification protocol for IEEE 802.11 wireless networks," in Proc. IEEE International Conf. Focusing Broadband Commun., Networks, Systems (BROADNETS'04), 2004.

[5] R. Garces and J. J. Garcia-Luna-Aceves, "Collision avoidance and resolution multiple access for multichannel wireless networks," in Proc. IEEE International Conf. Computer Commun. (INFOCOM'O0), Mar. 2000.

[6] A. Nasipuri, J. Zhuang, and S. R. Das, "A multichannel CSMA MAC protocol for multihop wireless networks," in Proc. Wireless Commun.Networking Conf. (WCNC'99), 1999.

[7] N. Choi, Y. Seok, and Y. Choi, "Multi-channel MAC protocol for mobile ad hoc networks," in Proc. IEEE Veh. Technol. Conf. (VTC'03), 2003.

[8] H. Zhai, J. Wang, and Y. Fang, "DUCHA: a dual-channel MAC protocol for mobile ad hoc networks," IEEE Trans. Wireless Commun., vol. 5, no. 11, Nov. 2006.

[9] S. L. Wu, C. Y. Lin, Y. C. Tseng, and J. P. Sheu, "A new multichannel MAC protocol with on-demand channel assignment for multihop mobile ad hoc networks," in Proc. International Sym. Parallel Architectures, Algorithms Networks (ISPAN'00), 2000.

[10] J. So and N. Vaidya, "Multichannel MAC for ad hoc networks: handling multichannel hidden terminals using a single transceive," in Proc. ACM International Sym. Mobile Ad Hoc Networking Computing (MobiHoc'04), 2004.

[11] J. Shi, T. Salonidis, and E. W. Knightly, "Medium access control: starvation mitigation through multi-channel coordination in CSMA multi-hop wireless networks," in Proc. ACM International Sym. Mobile Ad Hoc Networking Computing (MobiHoc'06), 2006.

[12] P. Bhagwat, P. Bhattacharya, A. Krishna, and S. K. Tripathi, "Enhancing throughput over wireless LANs using channel state dependent packet scheduling," in Proc. IEEE International Conf. Computer Commun. (INFOCOM'96), 1996.

[13] J. Wang, H. Zhai, Y. Fang, J. Shea and D. Wu, "OMAR: utilizing multiuser diversity in wireless ad hoc networks," IEEE Trans. Mobile Computing, vol. 5, no. 12, pp. 1764-1779, Dec. 2006.

[14] H. Zhai and Y. Fang, "Physical carrier sensing and spatial reuse in multirate and multihop wireless ad hoc networks," in Proc. IEEE International Conf. Computer Commun. (INFOCOM'06), Apr. 23-29, 2006

[15] H. Zhai and Y. Fang, "Impact of routing metrics on path capacity in multi-rate and multi-hop wireless ad hoc networks," in Proc. 14th IEEE International Conf. Network Protocols (ICNP'06), Nov. 12-15, 2006.

[16] G. Bianchi, "Performance analysis of the IEEE 802.11 distributed coordination function," IEEE J. Select. Areas Commun., vol. 18, no. 3, pp. 535-547, 2000.

[17] T. S. Rappaport, Wireless Communications: Principles and Practice. Prentice Hall, 1999.

[18] http://research.microsoft.com/mesh/

[19] [Online] Available: http://www.strixsystems.com

[20] [Online] Available: http://www.meshdynamics.com/ 